\title{
Cytokines in umbilical cord blood-derived cellular product: a mechanistic insight into bone repair
}

\author{
Mukta S Sane ${ }^{\ddagger} 1$, Neha Misra ${ }^{\ddagger}{ }^{1}$, Omid Mohammad Mousa ${ }^{1,2}$, Steve Czop ${ }^{3}$, Huiyuan Tang ${ }^{1}$, \\ Larry T Khoo ${ }^{4}$, Christopher D Jones*,1,3 \& Soumyajit Banerjee Mustafi**,1 \\ ${ }^{1}$ Department of Research \& Development, Burst Biologics, Boise, ID 83705, USA \\ ${ }^{2}$ Biomolecular Research Center, Boise State University, Boise, ID, 83725, USA \\ ${ }^{3}$ Department of Regulatory \& Medical Affairs, Burst Biologics, Boise, ID 83705, USA \\ ${ }^{4}$ The Spine Clinic of Los Angeles, Good Samaritan Hospital, University of Southern California, Los Angeles, CA 90017, USA \\ *Author for correspondence: Tel.: +1 888322 1191; Fax: +1 208629 5866; cjones@smart-surgical.com \\ **Author for correspondence: sbanerjee@smart-surgical.com \\ $¥$ Authors contributed equally
}

\begin{abstract}
Aim: Umbilical cord blood (UCB) finds frequent applications in regenerative medicine. We evaluated the role of cytokines present in a uniquely processed, UCB-derived cellular allograft product (UCBp). Materials \& methods: Luminex multiplex assay and standard cell biology methods were employed. Results: Study with allografts from 33 donors identified 44 quantifiable cytokines in the UCBp derived conditioned media (CM). The UCBp-CM elevated proliferation and migration rates of mesenchymal stem cells (MSCs) and bone marrow stromal cells. Moreover, UCBP-CM induced secretion of VEGF-A and osteoprotegerin, which promoted angiogenesis of endothelial cells and positively influenced the osteogenic differentiation of MSCs, respectively. Conclusion: Cytokines in UCBp stimulate cellular processes important for bone regeneration, making UCBp an excellent candidate for potential applications in orthopedic procedures like bone non-union and spinal fusion.
\end{abstract}

First draft submitted: 7 August 2018; Accepted for publication: 4 October 2018; Published online: 22 October 2018

Keywords: angiogenesis • bone non-union • cell signaling • migration • osteogenic differentiation • proliferation • spinal fusion

In patients with compromised health, bone repair and remodeling present a clinical challenge for orthopedic surgeons, with the most common complication being non-union [1-4]. Treatment of bone non-union is critical in preventing progressive deformity, relieving persistent pain and subsequently achieving a total functional recovery [5]. Consolidation of fracture depends on mechanical as well as biological factors, ranging from local cell proliferation and differentiation to vascularization [6]. Over the last several decades, intensive research efforts have focused on developing new approaches and technologies to enhance these biological factors for fracture healing and successful spine fusion. Electrical stimulators, bone morphogenetic proteins and adult cellular tissue-based products harvested from cadaver bones have gained prominence in the treatment of bone non-union as alternate therapies [3]. In recent years, cell and gene therapies have garnered much interest from the scientific community. The field of musculoskeletal regenerative medicine has been revolutionized through a better understanding of mesenchymal stem cell (MSC) biology [7]. MSCs have extensive therapeutic application because of their ability to differentiate into structural tissues like bone and secrete trophic factors like osteogenic biomolecules that stimulate bone repair and regeneration $[8]$.

The beginning stages of fracture healing are often associated with local inflammation, followed by MSC recruitment, differentiation and angiogenic activation phase [9,10]. Apart from mobilizing the patient's own stem cells to the site of bone injury with pharmacologic interventions, clinicians often use 'nonmanipulated' cell preparations that are likely to contain a healthy pool of MSCs $[11,12]$. In the last couple of decades, several researchers have reported that umbilical cord blood (UCB) contains multipotent MSCs, which can differentiate into connective 
tissue with demonstrable clinical benefits in diseases not only limited to hematopoietic disorders [13-19]. There are several advantages for using UCB as a source for MSCs, such as the harvesting procedure of postnatal cord blood is noninvasive and does not pose ethical concerns, UCB cells have relatively low immunoreactivity $[14,20,21]$ and UCB-derived MSCs have high osteogenic potential $[19,22,23]$. Although often overlooked, UCB is also a rich source of cytokines and growth factors [24]. Some studies have attempted to correlate the presence of specific cytokines in UCB with neonatal disease [25]. Other studies have identified cytokines like PDGF, GM-CSF, etc., in the UCB plasma that may have therapeutic benefits during transplantation [26-28]. Cytokines are involved in different stages of bone repair. Interleukins, such as IL6, MCP-1 and MIP-1, help in the inflammatory phase. Specific cytokines act as 'homing factors' to recruit endogenous MSCs at the site of injury. VEGF, PDGF, FGF-2, etc., also play important roles in proliferation, migration, and differentiation of MSCs into osteoblasts [29]. However, there have been no in-depth studies to determine and quantitate the cytokines present in UCB-derived products (UCBps) that are currently available for clinical applications. It is important to note that without the specific cytokines that can help in neovascularization and/or recruitment of progenitor cells, even UCB-derived MSCs cannot deliver on the promise of successful tissue repair [30,31]. This is more relevant for bone non-union, which is commonly associated with damaged vascular supply with impaired access to important mediators at the site of injury. Cytokines send specific signals to the resident bone marrow (BM) MSCs (BM-MSCs), which are then stimulated to leave their niche to be recruited within the vasculature of the tissue for transmigration across the endothelium (homing) $[32,33]$. Homing of exogenous and endogenous MSCs at the site of injury is a major cornerstone in tissue regeneration, which is followed by proliferation and differentiation into mature bone cells [34-36]. Therefore, the full potential of UCB for bone regeneration can only be harnessed if both multipotent stem cells and cytokines derived from UCB are efficiently formulated in a cryopreserved allograft for therapeutic interventions.

Cord blood banking has guidelines to ensure donor suitability and quality of cord blood used for transfusion/transplantation. Total nucleated cells and $\mathrm{CD} 34^{+}$cell population are the key parameters for selection and eligibility of cord blood units for transplantation [37]. In contrast, the current industry standard for manufacturing clinically usable products derived from cord blood is not well defined and involves procurement of cord blood, followed by recovery and collection of the nonerythroid subsets of cells [38], which are cryopreserved until application [39]. The quality of the final cord blood-derived product is therefore dependent on two major factors: donor variability (maternal factors) and processing methods used to manufacture, package and store the product. Mononucleated cell (MNC) count of the final product is a generally accepted parameter to determine overall quality of such commercially available products [40,41]. However, the lack of any other reliable parameters to determine regenerative potential and biological efficacy makes it extremely difficult to predict the clinical outcomes of such products.

The current study is aimed at determining the bone regenerative potential of a cellular allograft uniquely processed from UCB to optimize high MNC count and to enrich cytokine content. We hypothesized that if optimally processed, the final UCB-derived product could be significantly enriched with a broad array of cytokines. Subsequently, after application to the site of bone injury, the product could alter the tissue microenvironment and activate the residing host cells to participate in various stages of bone regeneration. We performed a first-of-its-kind profiling of 33 donors to determine the mean concentration of 44 cytokines present in this UCB-derived product. Using in vitro model systems, we further provide evidence to establish the pro-proliferative, pro-migratory, proangiogenic and pro-secretory potential of conditioned media (CM) obtained from a UCB-derived product. Taken together, our data provide insights into the molecular mechanism of action of this UCB-derived product currently undergoing clinical studies (NCT03064802 and NCT03077256) and being used for treating spinal fusion or bone non-unions.

\section{Materials \& methods UCB-derived product}

UCB obtained from consenting donors undergoing full term cesarean birth was processed by the patent pending method per the FDA's regulatory guidelines. All products were tested for MNC viability and microbial contamination prior to use.

\section{Primary \& secondary CM}

Primary CM was prepared from the UCBp (available from Burst Biologics, Boise, ID 83705, USA under the brand name BioBurst Fluid). The product was used directly or first sonicated (10 s pulse three-times on ice) and then 
diluted with basal (serum free) MSC media to 1:5 ratio, incubated at $37^{\circ} \mathrm{C}$ for $48 \mathrm{~h}$, and centrifuged at $1500 \times g$ for $5 \mathrm{~min}$. The supernatant was collected, sterile filtered and stored at $-80^{\circ} \mathrm{C}$ until further use. The $\mathrm{CM}$ was further diluted (1:1) with appropriate media for downstream experiments. Secondary CM was prepared by treating cells for $36 \mathrm{~h}$ either with the primary CM (pooled from 3-5 donors) diluted 1:1 with appropriate cell specific basal (serum free) culture media or with basal media only. The media were collected, centrifuged at $1500 \times g$ for 5 min, sterile filtered and further used in downstream assays.

\section{Cells \& tissue culture media}

HS-5 (BM stromal cells, CRL-11882), HS-27a (BM stromal cells, CRL-2496), BM-MSCs (BM-derived MSCs, PCS-500-012) and human umbilical vein endothelial cells [HUV-ECs], CRL-1730) were all purchased from ATCC (Manassas, VA, USA). HS-5 and HS-27a were cultured in DMEM and RPMI (VWR), respectively, supplemented with 10\% FBS (VWR, Radnor, PA, USA). BM-MSCs were cultured in the basal media (ATCC, PCS-500-030) supplemented with growth kit (ATCC PCS-500-041). HUV-ECs were cultured in EGM ${ }^{T M}$ Plus SingleQuots ${ }^{\circledR}$ (Lonza Cat \# CC4542). All cell lines were maintained in a humidified incubator at $37^{\circ} \mathrm{C}$ with $5 \% \mathrm{CO}_{2}$. Primary cell lines (BM-MSCs and HUV-EC) between 3 and 5 passages were used for all experiments.

\section{Cytokine measurement}

Cytokine concentration was measured from 33 donors using multiplex ProcartaPlex Panel (Thermo Fisher Scientific, CA, USA; EPX450-12171-901). Luminex xMAP magnetic-bead fluorescent immunoassays (Invitrogen) were run on MAGPIX ${ }^{\mathrm{TM}}$ and measurements were done as per the manufacturer's protocol. Primary CM limited to one freeze thaw cycle was used. Basal (serum free) MSC media served as baseline values for the assay. For each standard, percent recovery values outside $90-110 \%$ were invalidated using xPonent Analysis software and concentration of cytokines were calculated using a standard curve with $\mathrm{R}^{2} \geq 0.9$. VEGF-A and osteoprotegerin (OPG) concentration was measured in secondary CM using ProcartaPlex Kit (Thermo Fisher Scientific EPX01A-10277-901 and EPX42010200-901). Values were normalized to concentration of cytokines present in the basal MSC culture media.

\section{Cell proliferation assay}

Cells were seeded at a density of 5000 cells/well of 96-well plate and incubated overnight. Cells were washed with phosphate-buffered saline (PBS) and starved for $8 \mathrm{~h}$. Poststarvation, wells were replenished with complete media or primary CM diluted 1:1 with cell specific serum free culture media and incubated for additional $48 \mathrm{~h}$. The amount of DNA in each cell remains constant for a given cell line or cell type, so assays based on DNA content provide an accurate and simple measure of cell number. After $48 \mathrm{~h}$, cell proliferation was analyzed by measuring DNA content using CyQUANT Cell Proliferation Kit (Thermo Fisher Scientific, CA, USA) using manufacturer's protocol. Experiment was repeated twice using primary $\mathrm{CM}$ from 3 to 6 different donors and the values were normalized to the basal media (negative control).

\section{Scratch assay}

$2 \times 10^{4}$ cells/well were plated on a 48 -well plate and incubated overnight. After $8 \mathrm{~h}$ of serum starvation, a scratch was created at the center of the well using a $20 \mu \mathrm{l}$ micropipette tip. The cells were then washed twice with PBS and treated for $12 \mathrm{~h}$ with primary CM diluted 1:1 with cell specific basal media. Cells were washed twice in PBS to remove the debris before imaging. Images of the same scratched region were taken immediately after wounding and again at termination of experiment. At least three random fields were micrographed for each experimental condition. The number of cells that migrated across the scratched boundary was quantified using ImageJ. Relative migration was determined by evaluating the fold increase in the number of cells migrated upon treatment with conditioned/complete media when compared with untreated controls (serum-free media). Experiment was repeated twice using primary CM from 3 to 6 different donors.

\section{Transwell migration assay}

Cells were serum starved $8 \mathrm{~h}$ before performing the experiment. Transwell inserts were coated with $10 \mu \mathrm{g} / \mathrm{ml}$ of fibronectin (Sigma-Aldrich, MO, USA) and left overnight for surface adsorption. Cells were resuspended in serum-free basal media and $2 \times 10^{4}$ cells were added to the upper chamber of a 24 -well, $8 \mu \mathrm{m}$ pore membrane. Complete media, basal media or primary CM diluted 1:1 in cell specific basal media were added to the lower chamber as a chemoattractant. After $16 \mathrm{~h}$, cells on the upper surface were removed with a cotton swab and the 
remaining cells on the lower surface were fixed in 5\% glutaraldehyde (Sigma-Aldrich) and stained with $1 \%(\mathrm{w} / \mathrm{v}$ ) crystal violet stain (Sigma-Aldrich). Cells were imaged at $4 \times$, and imaged cells from ten random fields were used for quantification. Migration experiments were repeated twice using primary CM from 3 to 6 different donors and the values were normalized to the basal media (negative control).

\section{Spheroid sprouting assay}

For spheroid formation, $2 \times 10^{4} \mathrm{HUV}$-EC cells/well were plated on an ultralow binding round bottom 96-well plate for $48 \mathrm{~h}$. Subsequently, spheroids were collected and overlaid on plates coated with $200 \mu \mathrm{l}$ of 3D Collagen Cell Culture with reduced growth factors (MilliporeSigma, MA, USA) and supplemented with secondary CM for $24 \mathrm{~h}$. After $24 \mathrm{~h}$, individual wells were scanned, and spheroids were imaged by EVOS FL Auto 2 cell imaging system. Images were analyzed using Wimasis to determine total sprout number, cumulative sprout length and average sprout length.

\section{Tube formation assay}

For the tube formation assay, HUV-EC cells were seeded at density of $2 \times 10^{4}$ cells dispersed in secondary CM with either allograft or serum-free secondary CM in a 24-well plate coated with $200 \mu \mathrm{l}$ of Matrigel Basement Membrane Matrix (Thermo Fisher Scientific, PA, USA). Within 4-6 h of plating, wells were imaged by EVOS FL Auto 2 cell imaging system. Images were captured from five random microscopic fields for capillary-like structures and analyzed using ImageJ to calculate total tube length, total branching points, total tubes and total loops.

\section{Osteogenic differentiation assay}

The differentiation of BM-MSCs to osteoblast cells was evaluated as a function of alkaline phosphatase (ALP) activity or determining mineralization by Alizarin Red staining. Briefly, BM-MSCs were grown on 48-well plates in complete medium until $85 \%$ confluency. The medium was replaced after $72 \mathrm{~h}$ with $S$ temPro ${ }^{T M}$ osteogenic differentiation medium (Gibco/Fisher Scientific, PA, USA) that was either diluted with primary CM (1:5 respectively) or serumfree basal media (1:5). All the treatment groups received fresh media after every 3 days, until the experiment was terminated after 21 days. ALP activity was measured using an Alkaline Phosphatase Assay Kit (Abcam, MA, USA, ab83369) according to the manufacturer's protocol. For Alizarin Red Staining, cells were fixed with $70 \%$ cold ethanol for $30 \mathrm{~min}$ and then stained with $1 \%$ Alizarin Red solution for $10 \mathrm{~min}$ at room temperature. The cells were washed with milliQ water and air dried and images were captured from at least ten random fields.

\section{Data analysis \& statistics}

All experiments, unless otherwise stated, were repeated independently three times. Data are expressed as mean \pm standard deviation or standard error of the mean as indicated. Student's t-test was used for statistical analysis between two groups. Dunnett's test was used to compare among three or more groups. Statistical significance was set at $\mathrm{p}<0.05$.

\section{Results}

Cytokine profile of the UCBp

Cytokines are now considered to be an important cornerstone in regenerative medicine. So, we investigated if cytokines relevant to regeneration can be detected in the UCBp. UCBp obtained from 33 donors was diluted 1:5 in serum-free medium (SFM) and incubated for $48 \mathrm{~h}$ without stimulation. The concentration of 44 different extracellular cytokines in the UCBp-derived CM (UCBp-CM) was determined by Luminex MAGPIX ${ }^{T M}$ Assay. Growth factors (PDGF-BB, EGF), neurotrophic factors (BDNF, bNGF), angiogenic factors (VEGF) and pro- and anti-inflammatory chemokines with CCL (CCL2, CCL3, CCL4, CCL5, CCL11) and CXL (CXCL1, CXCL10, CXCL12) motifs were quantified (Figure 1A). The concentration of cytokines (mean \pm SEM) ranged from approximately $2 \mathrm{pg} / \mathrm{ml}$ to $13 \mathrm{ng} / \mathrm{ml}$. We found $40 \%$ of the total quantified cytokines had a concentration greater than approximately $250 \mathrm{pg} / \mathrm{ml}$ (Figure 1A). Concentration of the total VEGF (VEGF-A and VEGF-D) was $624.23 \pm 81 \mathrm{pg} / \mathrm{ml}$. EGF, a strong mitogen for ectodermal, mesodermal and endodermal cells, recorded a concentration of $234.48 \pm 13.63 \mathrm{pg} / \mathrm{ml}$. The mean concentration of PDGF-BB, a potent chemotactic and mitogenic agent, was $2231 \pm 200.93 \mathrm{pg} / \mathrm{ml}$. SDF1 $\alpha$ (CXCL1), a chemoattractant of mononuclear cells, had a concentration of $932.99 \pm 51.5 \mathrm{pg} / \mathrm{ml}$. The concentration of CCL-motif chemokines varied between approximately $40 \mathrm{pg} / \mathrm{ml}$ (CCL3) and approximately $1000 \mathrm{pg} / \mathrm{ml}$ (CCL12). All of these 44 cytokines detected in the UCBp-CM 


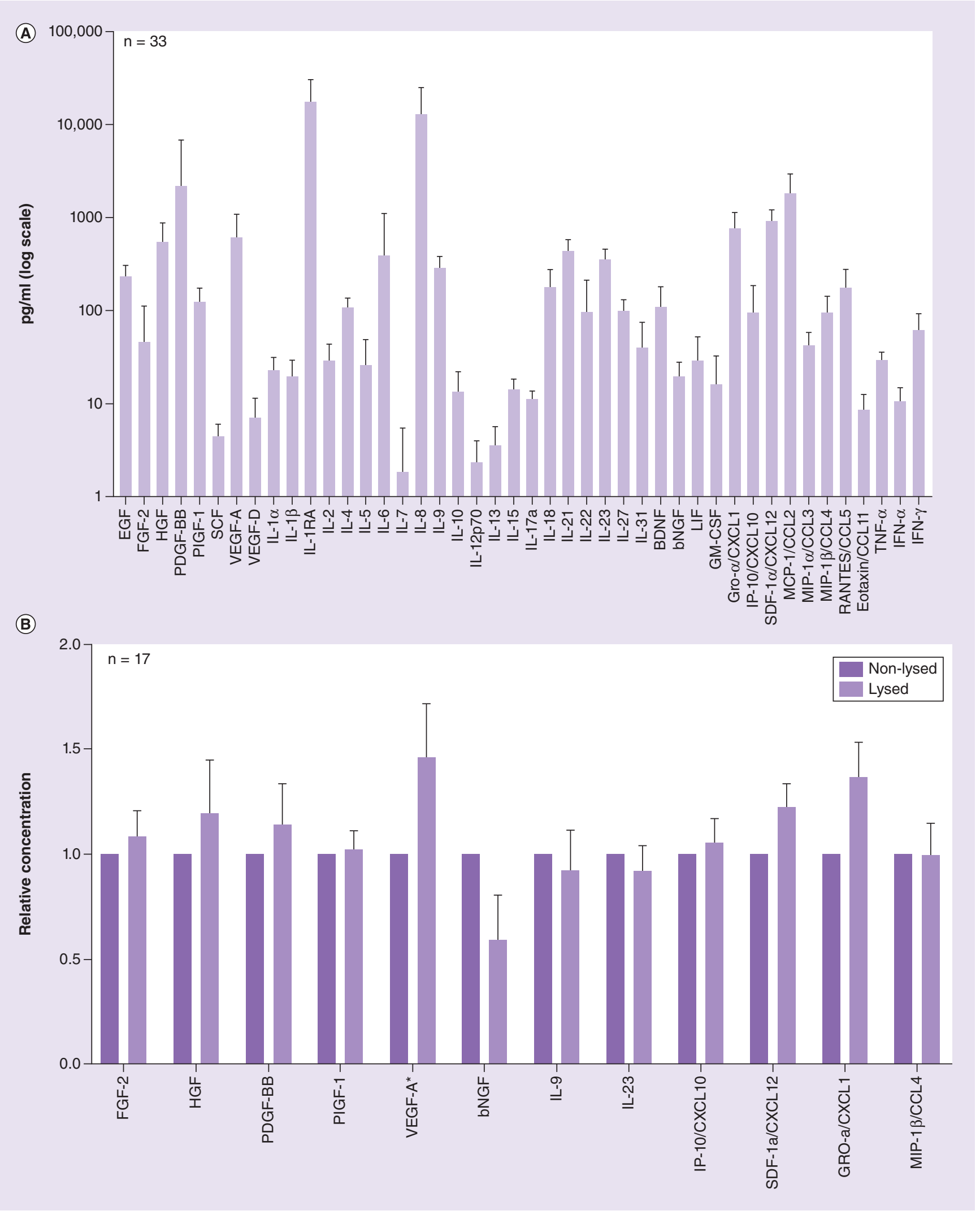

Figure 1. Cytokine profile of the umbilical cord blood-derived product. (A) Conditioned media (CM) was prepared using UCBp as described in the materials and methods section. Cytokine concentration was measured by immunoassays based on Luminex xMAP (multi-analyte profiling) technology in MAGPIX and expressed as mean values $( \pm S D)$ in picogram $/ \mathrm{ml}(\mathrm{n}=33$ donors). (B) Cytokine concentration in the CM derived from lysed (CM lysed) or intact (CM nonlysed) UCBp was determined. Values were compared between $\mathrm{CM}$ lysed and CM nonlysed obtained from same donor and expressed as mean ( \pm SEM) relative fold change (17 donors). Experiments were run in triplicates and * $p<0.05$ was considered statistically significant. 
directly or indirectly influence the process of bone repair (Supplementary Table 1). Hence, these data provide the first line of evidence that UCBp is enriched with cytokines that may trigger overlapping autocrine and paracrine signaling pathways to target MSCs, BM stromal cells and endothelial cells that regulate immune responses, inflammation, tissue remodeling and cellular differentiation.

The cytokines measured in the CM may be actively produced and secreted from the cellular component of the UCBp during the $48 \mathrm{~h}$ incubation period, or they may come from the noncellular component of UCBp and from cells in UCBp acting merely as reservoirs of these cytokines. To delineate between these two possibilities, UCBp obtained from the same donor was either incubated, as previously described, or sonicated to lyse the cells prior to incubation. Twelve cytokines were selected from a pool of 44 cytokines described in Figure 1A, based on their biological functions in healing bone non-union and spinal fusion (Supplementary Table 1). The concentration of these 12 cytokines in each pair (UCB-CM lysed and UCBp-CM nonlysed) was analyzed and compared across 17 donors (Figure 1B). The concentration between lysed and nonlysed products was not statistically different for any of the tested cytokines, except VEGF-A. VEGF-A concentration was 1.4-fold higher in CM lysed compared with $\mathrm{CM}$ nonlysed. If the cytokine concentration of CM was dependent on the intact cells in UCBp, then we would have expected a significantly reduced concentration of cytokines in the CM derived from the lysed UCBp. On the contrary, although statistically insignificant, a general trend noted was that the cytokine concentration in CM lysed was slightly higher than that of CM nonlysed. We speculate that the cellular components of the UCBp act as reservoirs of the intracellular and membrane bound cytokines and growth factors which are released by lysis in UCBp-lysed CM or by autolysis and/or passive secretion during $48 \mathrm{~h}$ incubation in serum free UCBp-CM.

\section{UCBp induces cell proliferation}

Damaged cells are replaced through routine cell proliferation. The regenerative potential of UCBp was therefore studied on BM-derived MSCs (BM-MSCs) and two immortalized BM-derived stromal cell lines (HS-5 and HS27a). After $48 \mathrm{~h}$ of treatment, SFM supplemented with UCBp-CM (1:1) induced higher proliferation in all three cell lines studied (Figure 2A-C) compared with cells cultured in SFM with no other exogenous factors. UCBp-CM treated HS-5 showed the highest fold increase ( $\sim$-fold) in proliferation, followed by HS-27a (3.36-fold) and BM-MSCs ( 2-fold). Cells that were cultured in the complete growth medium served as an internal reference of the normal cell proliferation rate. The proliferation rate of the UCBp-CM treated cells was higher than that of the group that was cultured in complete medium. We reasoned that the cocktail of cytokines, especially the growth factors (EGF, PDGF, HGF) present in UCBp-CM, transduce signals to promote entry of cells in the cell cycle, leading to higher cell proliferation. In addition to proliferation, the bone repair process entails migration of endogenous cells at the site of injury. We next investigated the chemoattractant potential of UCBP-CM.

\section{UCBp mediates migration \& chemotaxis}

To evaluate if UCBp influences the cell migration rate of BM-MSCs, HS-27a and HS-5, scratch assay and transwell migration assay were performed. After creation of the scratch, cells were treated for $12 \mathrm{~h}$ with SFM, SFM supplemented with UCBp-CM (1:1) or complete cell culture growth media. The experiment was terminated after $12 \mathrm{~h}$ to eliminate any confounding effect of cell proliferation that may arise from a longer period of incubation. Images of the scratch region were taken at initiation $\left(T_{0}\right)$ and at termination $\left(T_{12}\right)$ of the experiment. We determined that the migration rate of the cells in the presence of UCBp-CM was significantly higher $(p<0.05)$ than the cells treated with SFM. Compared with controls, the relative number of cells that migrated to the scratch area was sixfold higher for HS-27a (Figure 3B) and twofold higher for BM-MSCs (Figure 3D) when supplemented with UCBp-CM. Migration of the cells in 10\% FBS medium (complete) further demonstrates that the experimental parameters were optimum.

In addition to the 'wound healing' property as determined by the scratch assay, the potential of the UCBp as a chemo-attractant was specifically tested by transwell migration assay by using complete growth medium (10\% FBS), SFM or SFM supplemented with UCBp (1:1) as chemo-attractants. Chemotactic index was approximately twofold higher $(\mathrm{p}<0.05)$ for BM-stromal cell lines (HS-5 and HS-27a; Figure 4B \& D) and threefold $(\mathrm{p}<0.05)$ for the BM-MSCs compared with the SFM group (Figure 4F). These data (Figures $3 \& 4$ ) suggest that the trophic factors associated with UCBp are biologically active chemo-attractants and play a significant role in cell migration. As a part of the normal regeneration process, recruitment of resident cells is often followed by secretion of angiogenic factors that aid in the formation of new blood vessels. We next explored if the UCBp plays a similar role to influence angiogenesis. 


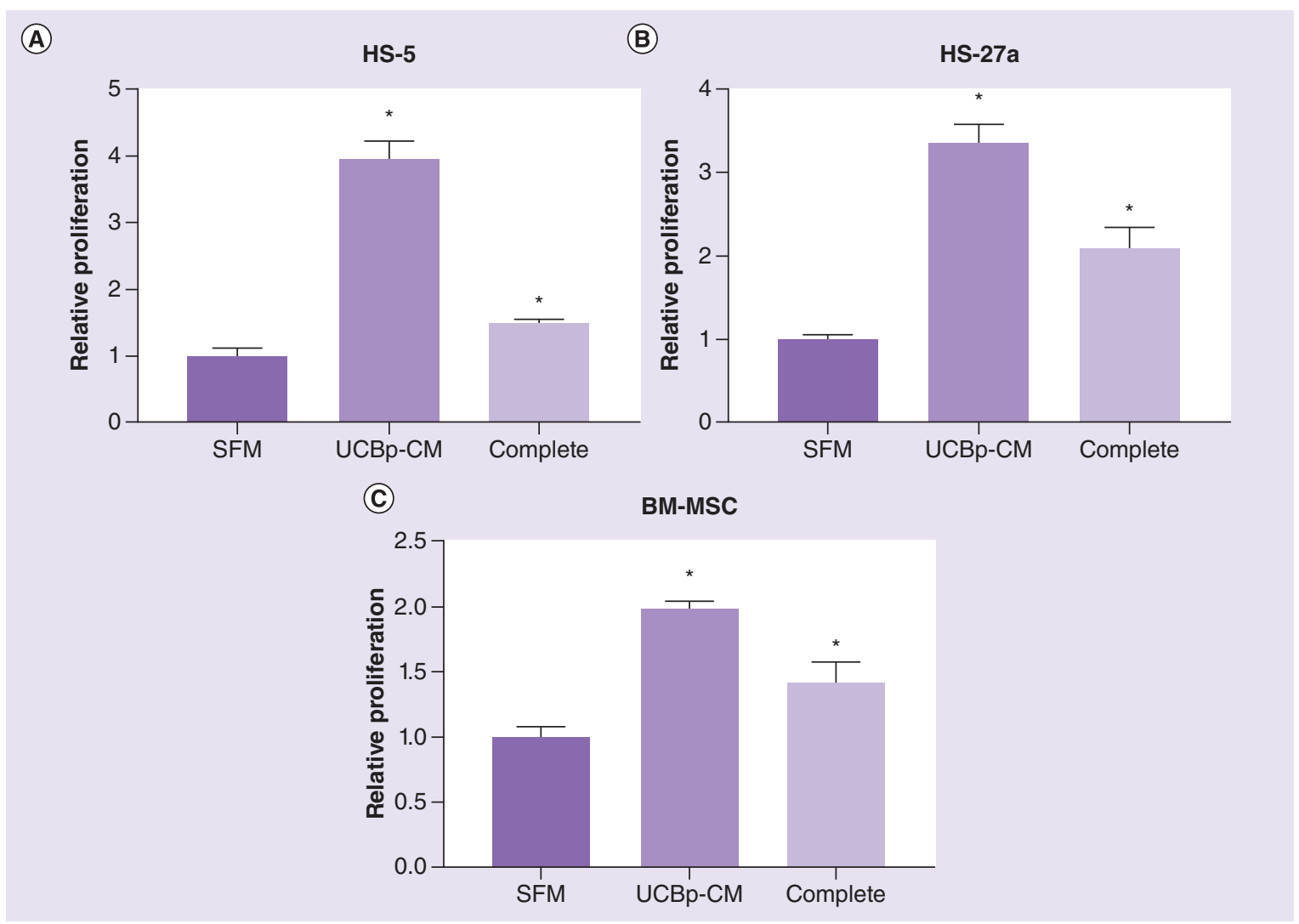

Figure 2. Umbilical cord blood-derived product-conditioned media induces cell proliferation in bone marrow derived stromal and mesenchymal stem cells. Proliferation rate was measured by fluorescence quantification of the total cellular DNA in HS-5 (A), HS-27a (B) and BM-MSCs (C) that were treated with SFM, UCBp-CM or complete media $(10 \% \mathrm{FBS})$. Data represented as mean \pm SD, relative to SFM treatment. Experiments were repeated with three different donors, at least in triplicates and * $p<0.05$ was considered statistically significant. CM: Conditioned media; FBS: Fetal bovine serum; MSC: Mesenchymal stem cell; SD: Standard deviation; SFM: Serum-free media; UCBp: Umbilical cord blood-derived product.

\section{UCBp influences cell secretory activity \& neovascularization}

MSCs and BM stromal cells can exert their therapeutic effects by secreting soluble or 'paracrine' factors to augment endogenous repair mechanisms. We tested if UCBp-CM can induce secretion of VEGF-A in HS-5, HS-27a and BM-MSCs. Cells were treated with SFM or SFM supplemented UCBp-CM (1:1) for $36 \mathrm{~h}$, and the concentration of VEGF in the secondary $\mathrm{CM}\left(\mathrm{CM}^{\mathrm{SFM}}\right.$ and $\left.\mathrm{CM}^{\mathrm{UCBP}}\right)$ was determined (Figure $\left.5 \mathrm{~A} \& \mathrm{~B}\right)$. The difference in VEGF concentration in the culture media between $\mathrm{T}=0 \mathrm{~h}$ and $\mathrm{T}=36 \mathrm{~h}$ represented the amount secreted by the cells in response to SFM or UCBp-CM. HS-5, HS-27a and MSCs treated with UCBp-CM secreted significantly higher VEGF-A (2.2-fold, 1.25-fold and 13.3-fold, respectively) compared with control SFM (Figure 5B). VEGF is a key regulator of angiogenesis. The formation of new blood vessels from pre-existing ones is mediated via two distinct pathways: splitting and sprouting. So, the pro-angiogenic potential of the secondary $\mathrm{CM}\left(\mathrm{CM}^{\mathrm{SFM}}\right.$ and $\left.\mathrm{CM}^{\mathrm{UCBP}}\right)$ was further evaluated by quantifying tube formation and spheroid sprouting of the HUV-EC (Figure 5C-H), as per the consensus guidelines for the interpretation of in vitro angiogenesis assays [42]. HUV-ECs that were grown in $\mathrm{CM}^{\mathrm{SFM}}$ did not form complex tubular structures, whereas the cells cultured in $\mathrm{CM}^{\mathrm{UCBp}}$ formed tubular networks with elaborate branching and nodes (Figure 5C-E). Tube formation was evident as early as $3 \mathrm{~h}$ following seeding onto the Matrigel matrix, and the structures were maintained for a minimum of $24 \mathrm{~h}$ (data not shown). Compared with controls $\left(\mathrm{CM}^{\mathrm{SFM}}\right)$, the total length of the tubes was significantly increased in the HUV-ECs treated with $\mathrm{CM}^{\mathrm{UCBp}}$ derived from HS-5 (Figure 5C), HS-27a (Figure 5D) and BM-MSCs (Figure 5E). However, the MSC-CM ${ }^{\mathrm{UCBp}}$ was less efficient at stimulating branching (branch length and branch points) compared with HS-5 and HS-27a derived secondary CM. Angiogenesis was also studied based on formation of capillary sprouts by HUV-EC spheroids (Figure 5F-H). CM ${ }^{\text {UCBP }}$ derived from HS-5 (Figure 5F), HS-27a (Figure 5G) and BM- 
(A) HS-27a

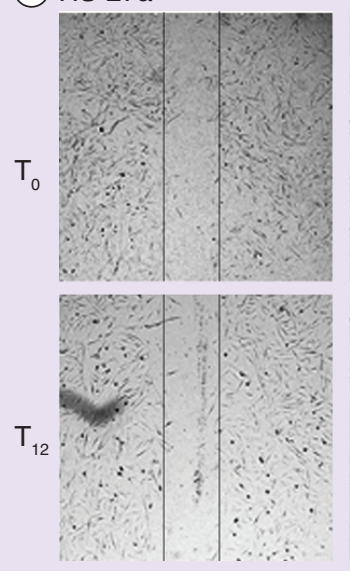

SFM

(C) BM-MSC

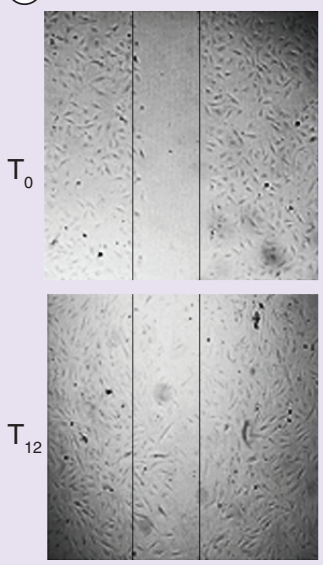

SFM

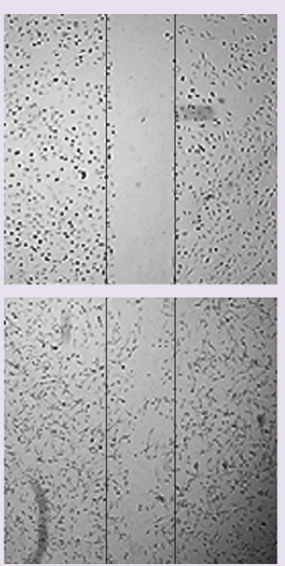

UCBp-CM

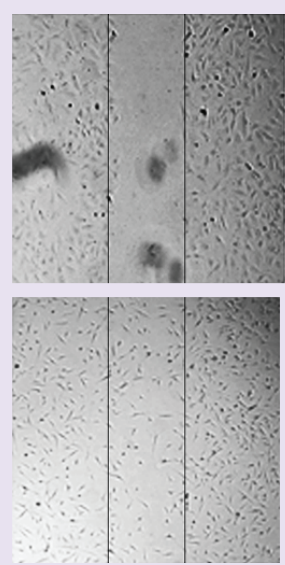

UCBp-CM

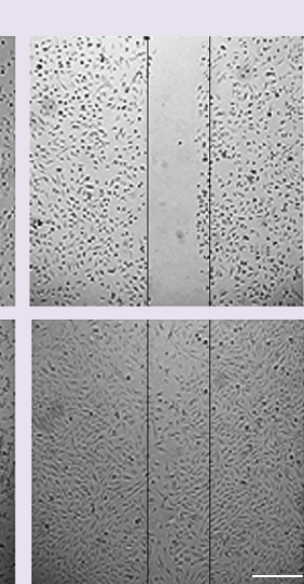

Complete

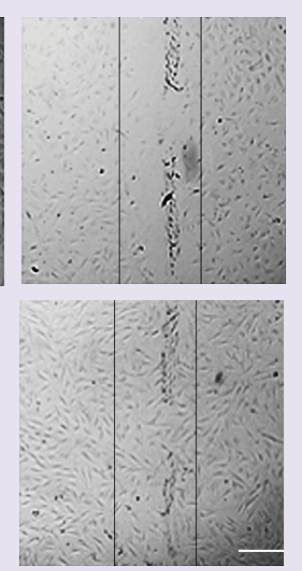

Complete
(B)

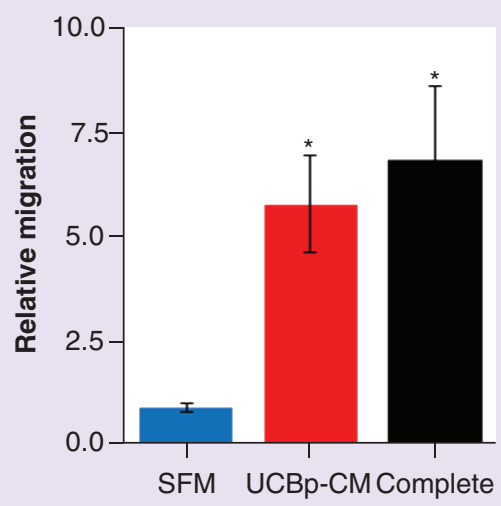

(D)

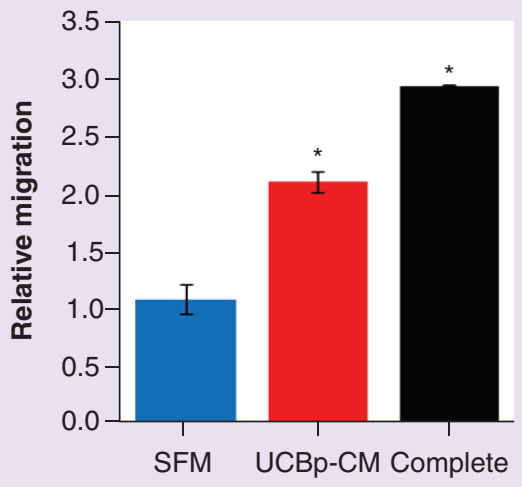

Figure 3. Umbilical cord blood-derived product-conditioned media enhances cell migration. Migration was evaluated with scratch assay in BM stromal cells; HS-27a (A \& B) and BM-MSCs (C \& D). Confluent monolayer of cells was serum starved for $8 \mathrm{~h}$, mechanically scratched and then treated with SFM, UCBp-conditioning media (UCBp-CM) or complete media for $12 \mathrm{~h}$. The scratch area was imaged at 0 and $12 \mathrm{~h}$, scale bar $=100 \mu \mathrm{m}$ (A \& C). Cell migration to the scratch area was quantified using ImageJ and represented as fold change (mean \pm SD) relative to SFM treatment. Experiments were repeated with three different donors, and quantification was based on 3-5 randomly imaged fields. ${ }^{*} p<0.05$ was considered statistically significant.

BM: Bone marrow; CM: Conditioned media; MSC: Mesenchymal stem cell; SD: Standard deviation; SFM: Serum-free media; UCBp: Umbilical cord blood-derived product.

MSCs (Figure 5H) induced significantly more capillary sprouting compared with $\mathrm{CM}^{\mathrm{SFM}}$. The cumulative sprout length and sprouting area were also significantly bolstered in HUV-EC spheroids incubated with CM from BBF treated HS-5, HS-27a and BM-MSCs compared with controls. The number of sprouts per HUV-EC spheroid also increased when treated with the $\mathrm{CM}^{\mathrm{UCBp}}$ derived from BM stromal cells (HS-5 and HS-27a), but not from BM-MSCs cells. These data on VEGF-A secretion and in vitro angiogenesis indicate a potential involvement of UCBp in new blood vessel formation. We next investigated if UCBp can also induce osteogenic differentiation of MSCs.

\section{UCBp induces osteogenic differentiation of MSCs}

New bone formation is often a critical balance between the physiologically opposite activities of osteoclasts and osteoblasts in which the RANK/RANKL/OPG axis plays an important regulatory role. We tested if treating HS-5, HS-27a and BM-MSCs with UCBp-CM induced secretion of OPG. Cells were treated with SFM or SFM 


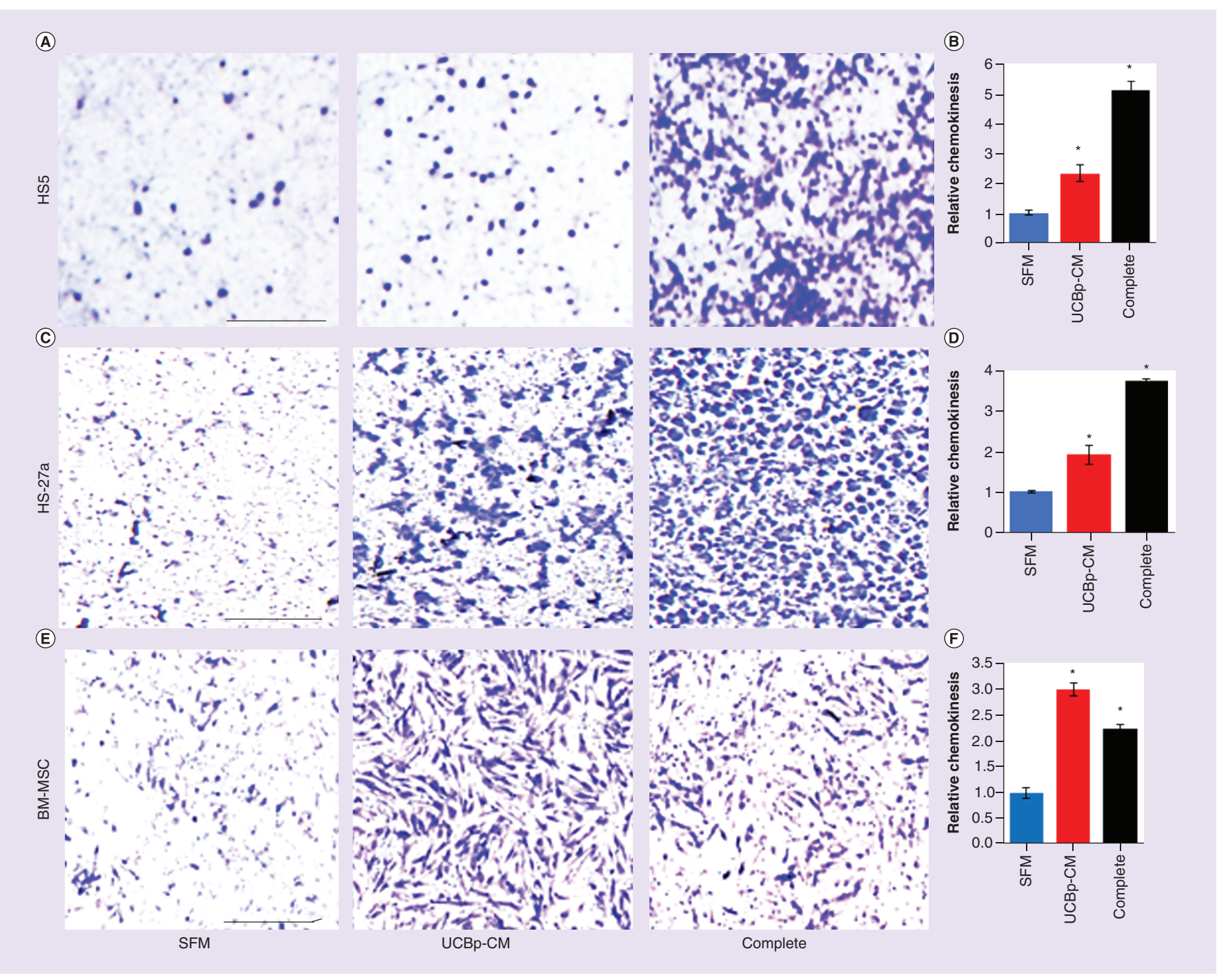

Figure 4. Umbilical cord blood-derived product-conditioned media induces chemotactic response. Chemotactic response of HS-5, HS-27a and BM-MSCs toward SFM, conditioning media obtained from product (UCBp-CM) or complete cell culture media (complete) was evaluated using a transwell system. Total number of cells adhered to the outer surface of the insert membrane were stained with crystal violet and micrographed, scale bar $=50 \mu \mathrm{m}(\mathrm{A}, \mathrm{C} \& \mathrm{E})$. Cells migrated per visual field were quantified using ImageJ (B, D \& F) and represented as relative chemokinesis (mean $\pm S D$ ) considering total cells migrated to SFM treatment as 1 . Experiments were repeated with three different donors, and quantification was based on three to five randomly imaged fields. * $p<0.05$ was considered statistically significant.

CM: Conditioned media; MSC: Mesenchymal stem cell; SD: Standard deviation; SFM: Serum-free media; UCBp: Umbilical cord blood-derived product.

supplemented UCBp-CM (1:1) as described earlier, and the concentration of OPG in the secondary CM $\left(\mathrm{CM}^{\mathrm{SFM}}\right.$ and $\mathrm{CM}^{\mathrm{UCBP}}$ ) was determined. HS-5, HS-27a and MSCs treated with UCBp-CM secreted approximately twofold to eightfold higher OPG compared with control SFM (Figure 6A).

Osteogenesis is also associated with robust ALP activity, which inevitably leads to mineralization of the neotissue. Osteogenic differentiation of the BM-MSCs was carried out in the absence and presence of UCBp-CM for 3 weeks, after which ALP activity and matrix mineralization were measured. BM-MSCs were cultured in one part of complete osteogenic differentiation media, either supplemented with four parts of UCBp-CM or four parts of SFM (Figure 6B). BM-MSCs cultured in presence of UCBp-CM demonstrated high ALP activity (Figure 6C) and significant mineralization (Figure 6D), indicating pronounced differentiation along the osteoblast lineage. ALP activity in BM-MSCs treated with UCBp can be detected spectrophotometrically as early as the tenth day after 
treatment (data not shown). ALP activity peaked at day 21 and was approximately 1.8 -fold higher than control. ALP activity increases the local concentration of inorganic phosphate required for biomineralization of bones and decreases the concentration of extracellular pyrophosphate known to inhibit bone mineralization. For detection of deposited mineralized matrix in the differentiating BM-MSCs cultures, the cultures were stained with Alizarin Red. Observed 21 days after treatment, the UCBp-grown cells displayed intense red color because of the calcium deposits when compared with control. Although BM-MSCs in both the control and experimental groups were cultured in the presence of diluted osteogenic differentiation media, the control group stained negative at day 21, despite having a moderate ALP activity. This observation is justified, given that ALP activity is an earlier event in osteogenic differentiation, and MSCs grown in undiluted osteogenic differentiation media may take up to 28 days to show matrix mineralization [43]. Hence, our data indicate that UCBp-CM significantly shortens the overall time required for osteogenic differentiation of MSC through plausible reprogramming of genes associated with bone mineralization.

\section{Discussion}

UCB has recently been added to the extensive list of cell sources for tissue engineering. Earlier, UCB was primarily considered for treating blood-related disorders and inherited metabolic diseases, owing to its enriched hematopoietic stem cells [44]. However, considering the rich pool of nonhematopoietic progenitor cells in UCB, cord blood finds potential clinical application in diverse fields from autism spectrum disorder [45] to regenerative orthopedics [46]. Cord blood-derived products available for regenerative applications lack well-defined parameters like reliable cell surface markers, colony forming unit counts and proven in vitro efficacy in cellular models. Physicians therefore

(A)

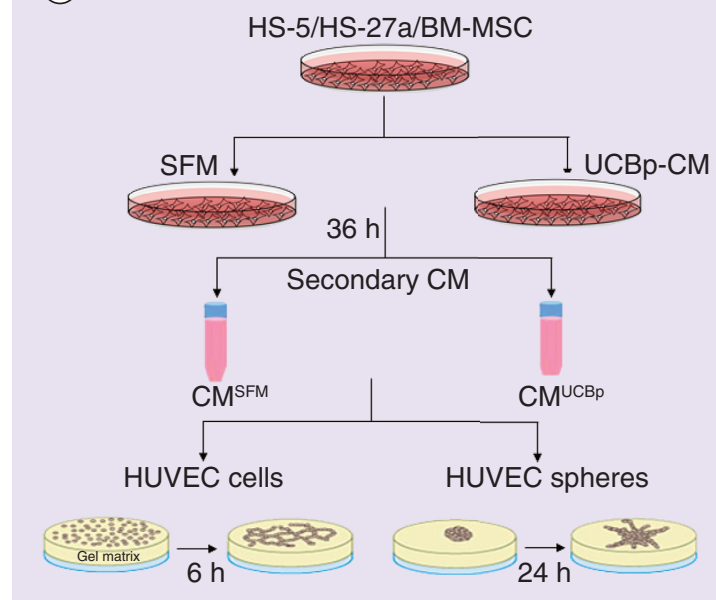

(B)

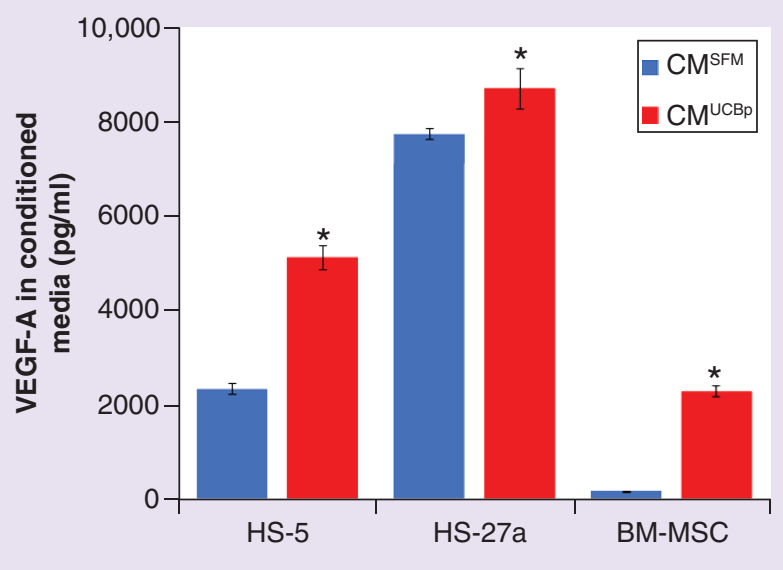

Figure 5. Umbilical cord blood-derived product-conditioned media mediated angiogenic response. (A) Schematic representation of the experimental design for preparation of secondary $\mathrm{CM}$ and subsequent in vitro angiogenesis assays (C-H) using human umbilical vein endothelial cells (HUV-EC). (B) Concentration of VEGF-A secreted by HS-5, HS-27a and BM-MSCs treated with UCBP-CM or SFM. (C-E) HUV-EC were cultured in Matrigel-coated plate in presence of $\mathrm{CM}^{\mathrm{SFM}}$ or $\mathrm{CM}^{\mathrm{UCBP}}$ obtained from HS-5 (C), HS-27a (D) or BM-MSCs (E). (C-E) Left-hand panels are representative photographs of the tube morphology formed after $4 \mathrm{~h}$ of treatment, scale bar $=100 \mu \mathrm{m}$. (C-E) Right-hand panels are the quantification of the tube morphology by ImageJ and represented as percent change (mean \pm SD) of the tube length, branching length and the branching point relative to $\mathrm{CM}^{\mathrm{SFM}}$ treatment. $(\mathrm{F}-\mathrm{H})$ Spheroids formed from HUV-EC cells were overlayed on 3D Collagen supplemented with either CM ${ }^{\text {SFM }}$ or CMUCBp (1:1) obtained from HS-5 (F), HS-27a (G) or BM-MSCs (H). (F-H) Left-hand panels are representative images of spheroid sprouting captured $24 \mathrm{~h}$ after treatment, scale bar $=50 \mu \mathrm{m}$. (F-H) Right-hand panels show cumulative sprout length, number of sprouts/spheroids and sprout area quantified by Wimasis and presented as mean pixels \pm SD. All experiments were performed three times. Quantification of tube morphology was done based on 3-5 randomly captured visual field images and sprouting morphology was quantified from an average of 3-6 spheroids per condition. * $p<0.05$ was considered statistically significant compared with SFM treatment group.

CM: Conditioned media; HUVEC: Human umbilical vein endothelial cells; MSC: Mesenchymal stem cell; SD: Standard deviation; SFM: Serum-free media; UCBp: Umbilical cord blood-derived product. 

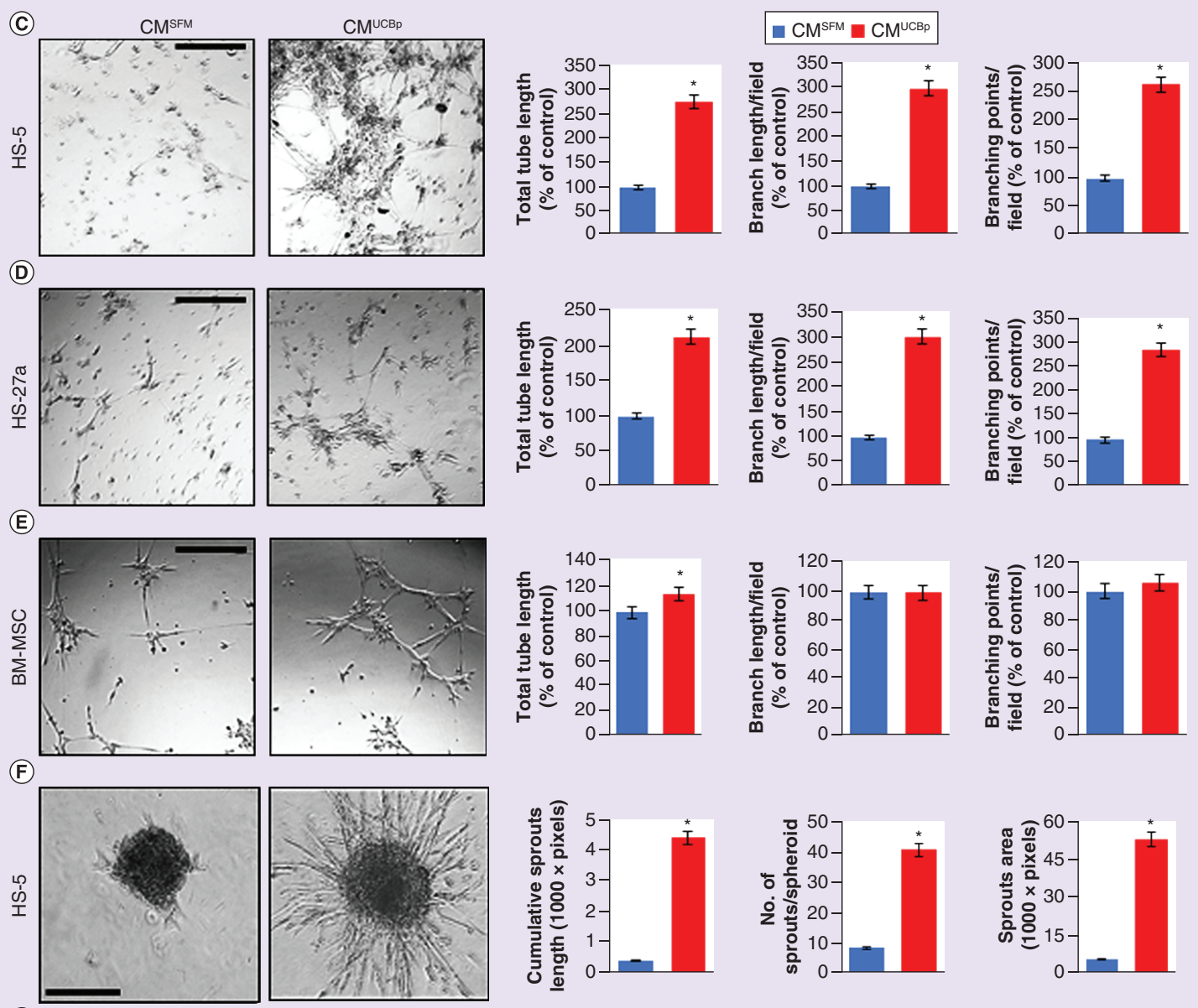

(G)
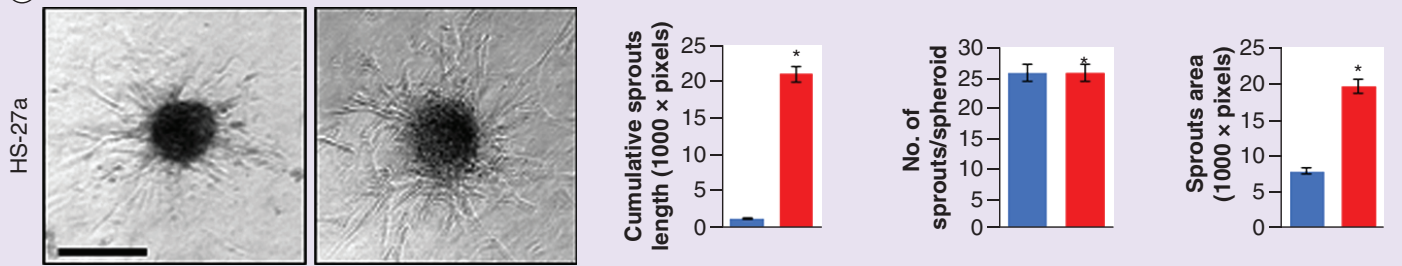

(H)
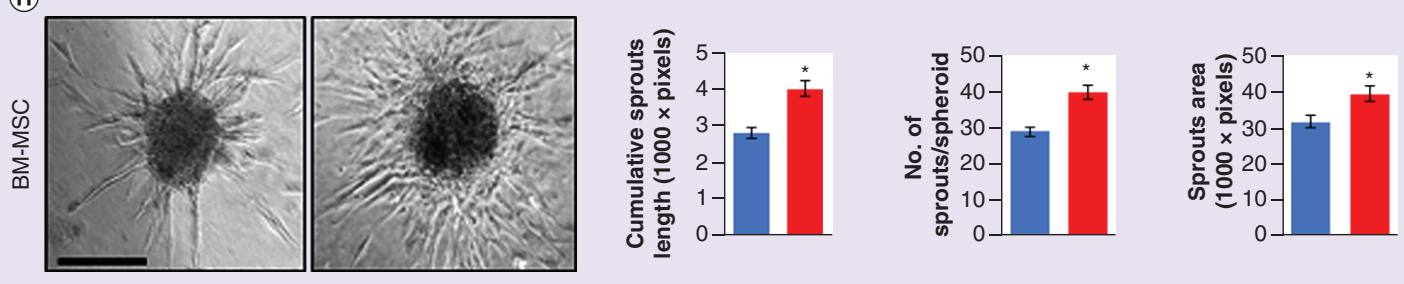

Figure 5. Umbilical cord blood-derived product-conditioned media mediated angiogenic response (cont.). (A) Schematic representation of the experimental design for preparation of secondary CM and subsequent in vitro angiogenesis assays (C-H) using human umbilical vein endothelial cells (HUV-EC). (B) Concentration of VEGF-A secreted by HS-5, HS-27a and BM-MSCS treated with UCBp-CM or SFM. (C-E) HUV-EC were cultured in Matrigel-coated plate in presence of $\mathrm{CM}^{\mathrm{SFM}}$ or CMUCBp obtained from HS-5 (C), HS-27a (D) or BM-MSCS (E). (C-E) Left-hand panels are representative photographs of the tube morphology formed after $4 \mathrm{~h}$ of treatment, scale bar $=100 \mu \mathrm{m}$. (C-E)

Right-hand panels are the quantification of the tube morphology by ImageJ and represented as percent change (mean \pm SD) of the tube length, branching length and the branching point relative to $\mathrm{CM}^{\mathrm{SFM}}$ treatment. (F-H) Spheroids formed from HUV-EC cells were overlayed on 3D Collagen supplemented with either $\mathrm{CM}^{\mathrm{SFM}}$ or $\mathrm{CM}^{\mathrm{UCBp}}(1: 1)$ obtained from HS-5 (F), HS-27a (G) or BM-MSCS (H). (F-H) Left-hand panels are representative images of spheroid sprouting captured $24 \mathrm{~h}$ after treatment, scale bar $=50 \mu \mathrm{m}$. (F-H) Right-hand panels show cumulative sprout length, number of sprouts/spheroids and sprout area quantified by Wimasis and presented as mean pixels \pm SD. All experiments were performed three times. Quantification of tube morphology was done based on 3-5 randomly captured visual field images and sprouting morphology was quantified from an average of 3-6 spheroids per condition. * $p<0.05$ was considered statistically significant compared with SFM treatment group.

CM: Conditioned media; HUVEC: Human umbilical vein endothelial cells; MSC: Mesenchymal stem cell; SD: Standard deviation; SFM: Serum-free media; UCBp: Umbilical cord blood-derived product. 


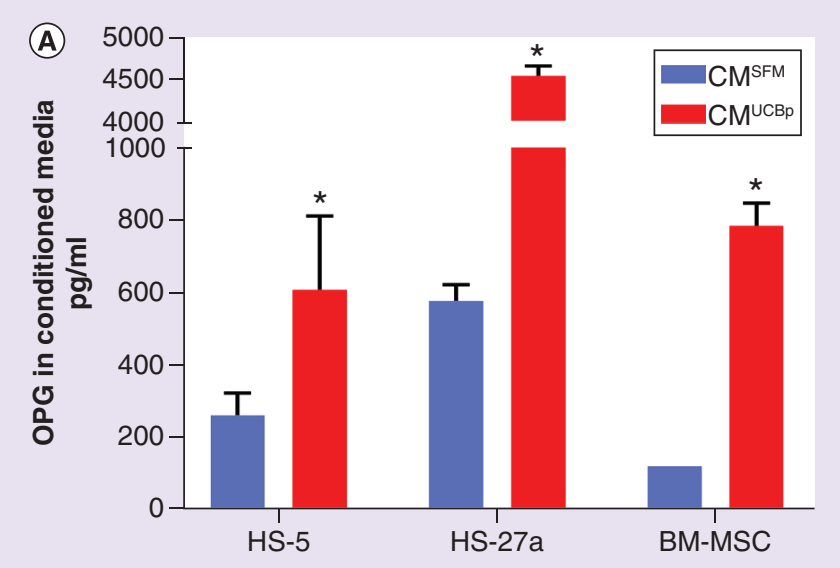

(B) $\quad M S C$
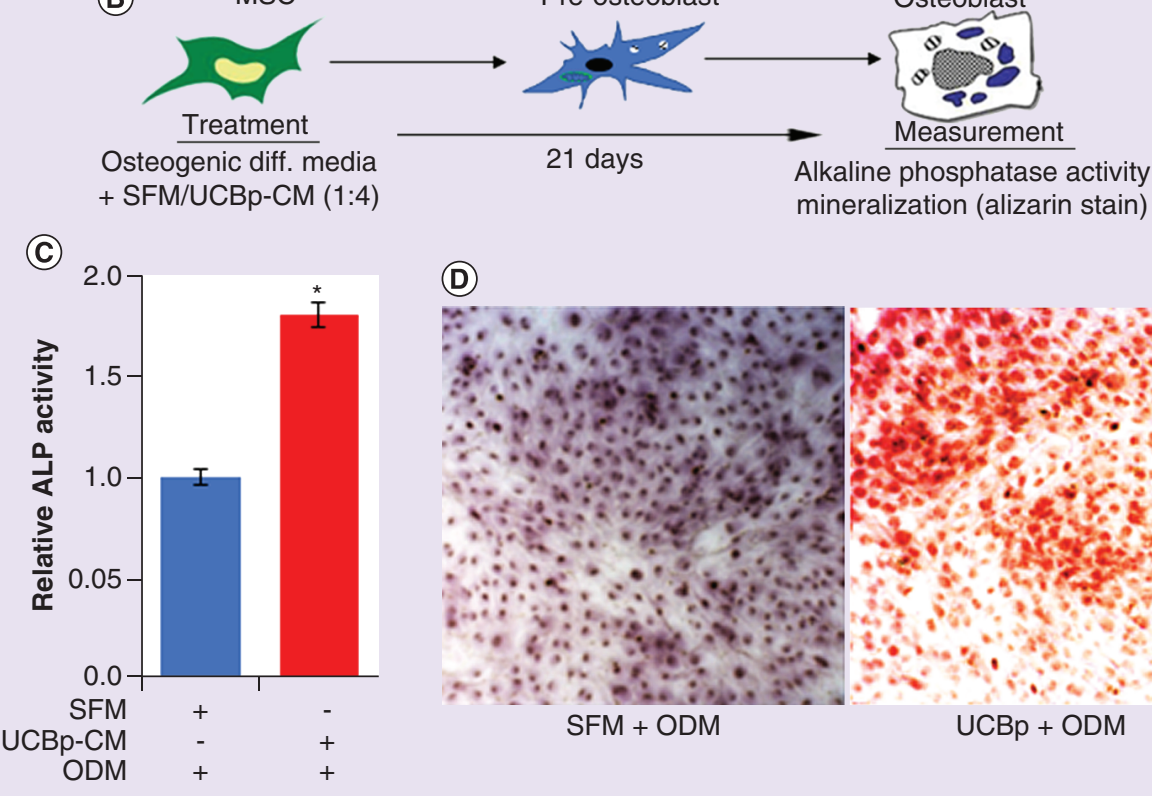

(D)

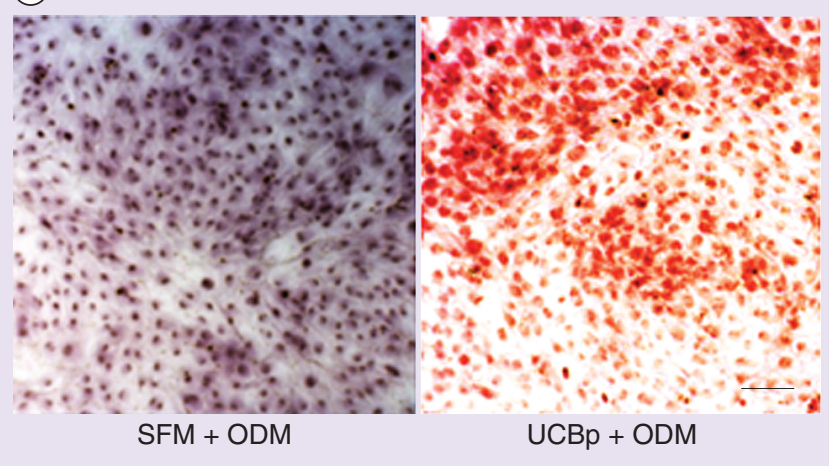

Figure 6. Umbilical cord blood-derived product induces osteogenic differentiation of bone marrow-mesenchymal stem cell. (A) Osteoprotegerin concentration was determined in the secondary conditioned media ( $\mathrm{CM}^{\mathrm{SFM}}$ or $\mathrm{CM}^{\mathrm{UCBp}}$ ) obtained in HS-5, HS-27a and BM-MSCs were expressed in $\mathrm{pg} / \mathrm{ml} \pm \mathrm{SD}$. (B) Schema summarizing treatment conditions and experimental timeline for osteogenic differentiation of MSC. (C \& D) After 21 days of differentiation in osteogenic medium diluted with UCBp-CM or SFM, BM-MSCs were accessed for the alkaline phosphatase activity (C) or mineralization by Alizarin red staining (D). Data are expressed as mean fold change \pm SD relative to MSCs cultured in SFM +ODM. Representative images are shown, scale bar $=200 \mu \mathrm{m}$. Experiments were repeated three times and in triplicates, and $\mathrm{p}<0.05$ was considered statistically significant.

ODM: Osteogenic differentiation media; BM: Bone marrow; CM: Conditioned media; MSC: Mesenchymal stem cell; SD: Standard deviation; SFM: Serum-free media; UCBp: Umbilical cord blood-derived product.

often rely on MNC counts or 'ill-defined' stem cell populations for ensuring clinical efficacy. However, these cell counts may not always correlate to clinical success. Previous research has shown that an absence of 'homing' factors in cell-based regenerative therapies has often led to limited recruitment of exogenous stem cells at the injury site [47]. So, in addition to stem cell restoration, the regenerative effects of tissue allografts depend on the paracrine action of cytokines that can also stimulate the residing host cells to take part in bone healing and regeneration. The challenge of developing a reliable UCB-derived tissue allograft therefore depends on recovering consistently high MNC yields during volume reduction and maintaining the inherent molecular heterogeneity and biochemical characteristics of UCB. 
Our study is aimed at understanding the comprehensive cytokine profile of a UCB-derived tissue allograft (UCBp, BioBurst Fluid) undergoing clinical studies for bone consolidation procedures like spinal fusion and foot and ankle surgery (NCT03064802 and NCT03077256). We provided direct evidence that UCBp is enriched with cytokines important for bone repair (Figure 1). The cytokine-rich composition of this product can be attributed to the careful processing of UCB that preserves both cellular and noncellular components (Figure 1A). Overall, our data suggest that rather than the cells in the UCBp, it is the acellular component of UCBP-CM that demonstrates strong therapeutic potentials (Figure 1B). This is in tune with a recent paradigm shift from MSC-based therapy to secretion-based therapy. The differentiation of exogenous stem cells into regenerating tissue may be a secondary effect. The cytokines secreted by these cells primarily mobilize and stimulate the residing host cells in the patient's body to help in the regenerative process [48]. More recently, MSC-derived exosomes and microvesicles are being examined for their potential role in a cell free MSC-based therapy [49]. Therefore, in the future, secretome based therapy might be a major cornerstone in regenerative medicine. Compared with cytotherapy, where malignant transformation during in vitro cell expansion or after transplantation is a potential concern, secretome based products would provide safer alternatives.

Apart from MSCs, BM stromal cells are also important for skeletal regeneration, as evident from successful clinical studies of autologous BM-derived stromal cells in non-union of bones, spinal fusion and osteoarthritis [50,51]. Both BM-derived MSCs and stromal cells responded favorably when treated with the UCBp-derived CM. Cell proliferation was significantly higher in the UCBp-CM treated BM-MSCs, HS-5 and HS-27a cells (Figure 2). Growth factors like PDGF, EGF, VEGF and FGF present in the UCBp (Figure 1; Supplementary Table 1) most likely activate the Ras-Raf-MEK-ERK and MAPK signaling cascades $[52,53]$ that consequently modulate cell cycle regulators and checkpoint proteins like cyclins D2, cyclin E and p27Kip to induce cell proliferation [54].

Cell homing (migration) of endogenous MSCs and BM stromal cells to the damaged bones and their subsequent participation in bone regeneration is a natural self-healing response [55]. Previous reports demonstrated that administration of the recombinant FGF, HGF and PDGF induce chemotaxis of the MSCs [56,57]. Preincubation with specific cytokines is also known to activate (SDF1/CXCL2/CXC) chemokine receptor 4 (CXCR) signaling, which leads to migration of BM-MSCs to bone injury site [58,59]. Stimulated mobilization and chemotaxis of the UCBp-CM treated MSCs and BM stromal cells as shown in Figures $3 \& 4$ can therefore be attributed to the biologically active chemotactic cytokines present in UCBp (Figure 1A; Supplementary Table 1).

Angiogenesis is important for bone regeneration as new blood vessels bring oxygen and nutrients to the metabolically demanding regenerating callus [60,61]. Clinical studies and animal models both show angiogenic induction by exogenous application of recombinant VEGF, FGF and PDGF [62]. Gerber $e$ al. noted that VEGF promotes capillary invasion, bone formation in growth plate and ECM remodeling in mice [63]. Eckardt et al. demonstrated that VEGF stimulates new bone formation in the atrophic non-union model by using torsional failure tests and callus size in rabbits [64]. We demonstrated that UCBp is enriched in proangiogenic factors like VEGF and PDGF (Figure 1A; Supplementary Table 1). Additionally, UCBp induced production of endogenous VEGF-A by BMMSCs and BM stromal cells, as shown in Figure 5B, possibly through the p38 mitogen-activated protein kinase (MAPK)-dependent pathway [65,66]. VEGF-A secretion by BM-MSCs and BM stromal cells also correlated to the formation of tubular networks and angiogenic sprouting by endothelial cells (Figure 5C). These data indicate a far-reaching impact of the UCBp's applications in bone injury. The UCBp acts as a dual advantage system by providing exogenous VEGF for immediate effect and initiating amplification of intrinsic angiogenic signaling for a more sustained benefit.

In addition to dependence on angiogenic pathways, a fundamental process of hard tissue biology and fracture healing involves endochondral ossification. The formation of cartilaginous callus at the fracture site is followed by chondrocytes maturing to hypertrophy, undergoing apoptosis and ultimately leading to new bone formation by invading osteoprogenitors. This resorption of the mineralized cartilage is regulated by well-orchestrated signaling pathways triggered by macrophage colony-stimulating factor, receptor activator of NF- $\mathrm{B}$ ligand (RANKL), OPG and TNF- $\alpha[67,68]$. Although we have not directly investigated the possible role of UCBp in chondrogenesis and endochondral ossification, several of the cytokines and growth factors that help in this process have been identified in UCBp. We have also seen that cells treated with UCBp-CM upregulated OPG secretion (Figure 6A). OPG inhibited osteoclast formation and $O P G$-deficient mice exhibit a decrease in total bone density and a high incidence of fractures. Using a mouse model, researchers have shown that OPG could impact bone healing and bone fusion, not only in spine fusion surgery, but also in the general area of bone healing from fractures [69]. The process 


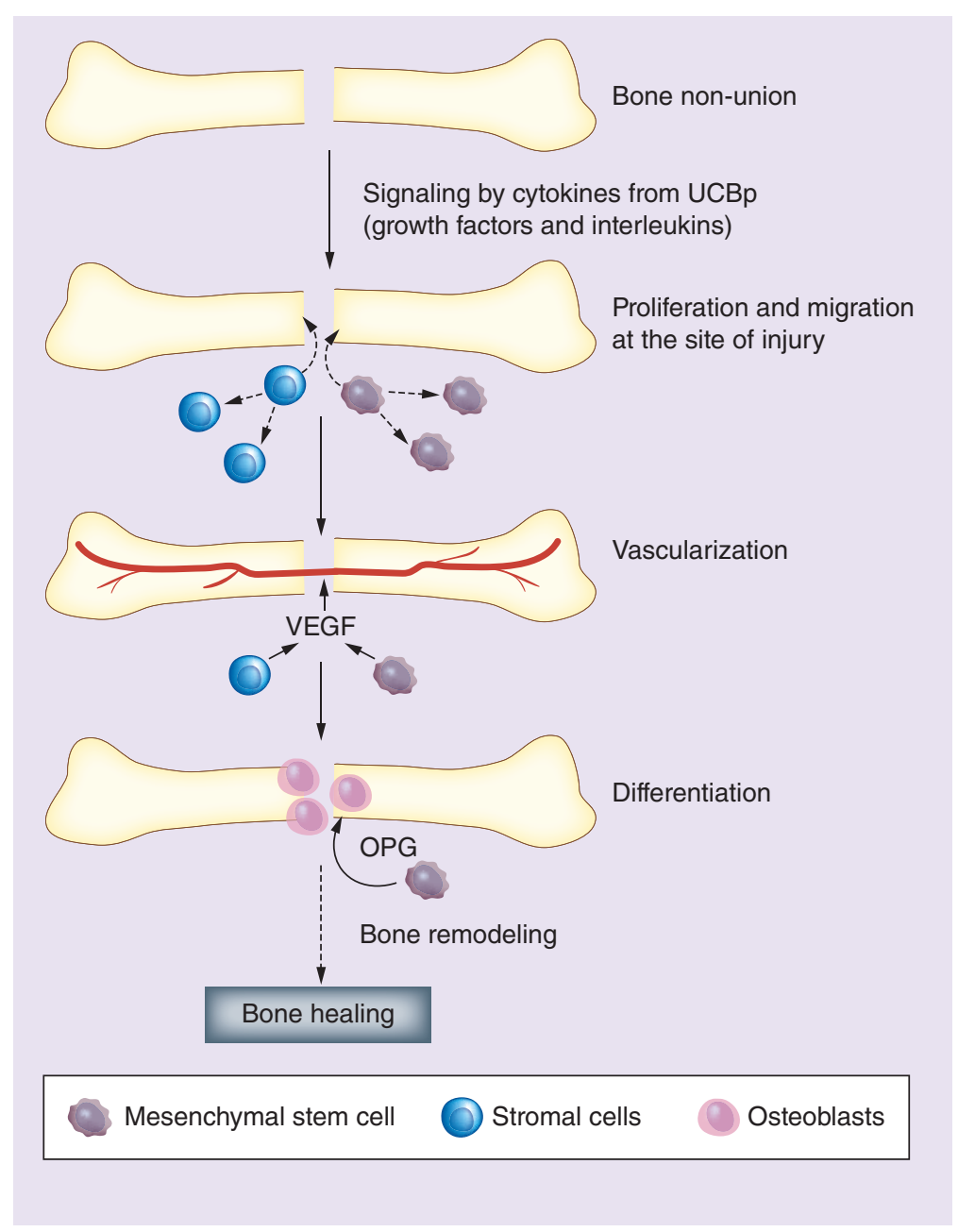

Figure 7. Model summarizing the molecular action of the umbilical cord blood-derived product in bone regeneration. Cytokines in UCBp initiate signaling pathways and activate residing BM stromal and mesenchymal stem cells to participate in bone regeneration. Homing factors in the UCBp help to recruit them at the site of injury and mitogenic factors support proliferation of the residing host cells. Proangiogenic factors like VEGF help in neovascularization, which brings in cells, nutrients and oxygen at the metabolically active site of tissue repair. Osteoinductive factors in the UCBP and induction of OPG help the recruited MSCs to differentiate into osteoblasts. This is followed by bone remodeling, leading to fracture repair or efficient spinal fusion.

BM: Bone marrow; MSC:

Mesenchymal stem cell; OPG:

Osteoprotegerin; UCBp: Umbilical cord blood-derived product.

of differentiation of progenitor cells to osteoblast lineage has been targeted for bone fracture and non-union management.

BMP-2 and BMP-7 are approved by US FDA to induce osteogenesis under pathological orthopedic conditions [70]. Other osteogenic factors currently in various stages of clinical study include VEGF, FGF2, PDGF2, LIF and interleukins (IL-6 family) [71]. As Figure 1 demonstrates, this allograft is enriched with many such osteoinductive factors which are important for bone repair and regeneration. Authors reported improved ossification in a mouse femur fracture model [72] and noted enhanced mineral density in a rat model by VEGF. In a canine tibial fracture model, injecting recombinant FGF-2 at the fracture site improved bone mineralization [73]. Our data on osteogenic differentiation of the BM-MSCs by UCBp (Figure 6) are therefore in complete agreement with the previous reports and underpin the interdependent and complex roles of the cytokines present in this UCBp which support osteogenesis.

\section{Conclusion}

We provide strong experimental evidence to establish that UCBp is enriched with cytokines and impacts cellular functions like proliferation, migration, differentiation, angiogenesis and paracrine signaling. These cellular processes are integral to bone regeneration. So, this in vitro study provides a plausible mechanistic explanation on how a UCB product can efficiently coordinate osteogenic cell recruitment, osteogenic differentiation/neovascularization and bone matrix production at the repair site to expediate healing in patients suffering from bone non-union, degenerative disc disorders or a high degree of spinal instability (Figure 7). Given the inherent multifactorial and multimodal nature of bone regeneration, one or even a few exogenous cytokines may only provide a short-term palliative relief, but not a sustained, curative outcome. In contrast, based on the efficiency of the method that 
processes UCB for UCB-derived clinical products, a broad range of cytokines can be preserved in a bioactive state and potentially applied for tissue regeneration. Thus, with the quantifiable levels of mitogenic, angiogenic and osteogenic factors in UCBp, it may provide a reliable alternative to application of the exogenous growth factors and ensure complete bone remodeling. Additionally, UCBp can counter the possible limitations of 'homing' in cellbased therapy by activating the exogenous and endogenous MSCs to be recruited at the site of injury. Altogether, our data establish the regenerative potential of the evaluated UCBp, making it an excellent candidate for treating orthopedic complications. Prospective clinical studies currently being conducted will further expand the scope of clinical application of this UCBp through informed outreach. Future investigations should include comparative analysis of similar products to decipher the roles of cytokines in bone regeneration.

\section{Translational perspective}

Stem cell-based therapy for the treatment of orthopedic diseases is arguably one of the most remarkable developments in the field of regenerative medicine. A better understanding of MSC biology and identification of MSCs in cord blood have added UCB to the sources of stem cells used for treatment of nonhematopoietic diseases. Most clinical studies on diseases like cerebral palsy and osteochondral lesions use in vitro expanded mononuclear cells which are isolated from UCB. Exogenous MSCs mostly get trapped in liver, spleen and lungs, and do not efficiently home to the targeted site. Therefore, any positive clinical outcomes of these clinical studies may be attributed to the signaling action of the secreted cytokines, rather than the cells themselves [74]. With the advancement of the processing methodology and preservation techniques, there are several commercially available UCB-derived allografts that have gained prominence in regenerative medicine applications. These allografts are often critiqued for their broadly variable clinical outcomes. Absence of universally accepted parameters to determine the regenerative potential and the current over-reliance on the total MNCs present in the allografts have been the major hurdles in full realization of the benefits of these allografts. To address this lacuna, comprehensive translation studies must be conducted in conjunction with comparative and prospective clinical studies that will focus on UCB-derived allografts in their entirety, and not just on isolated and expanded stem cells. Outcomes of two ongoing clinical studies on spinal fusion and foot-ankle surgery using UCBp as the only intervention will be correlated to the findings from this translation research to interpret the role of the cellular and noncellular components of UCB derived allografts in bone regeneration.

\section{Summary points}

- Umbilical cord blood (UCB) has long been used for allogenic transplantation in hematologic disorders because of its enriched pool of multipotent stem cells. However, with enhanced processing techniques, volume reduced and cryopreserved UCB-derived allografts are now routinely applied as adjunct treatments for regenerative therapy, including spinal fusion and bone non-union.

- Previous research and clinical studies on UCB-derived products in orthopedic procedures correlated positive clinical outcomes to the stem cell pool, but underestimated the possible roles of important cytokines, growth factors and immunomodulators present in UCB.

- Here, we investigated a uniquely processed and cryopreserved UCB-derived cellular allograft (UCBp) to determine its osteogenic capacity and bone regeneration potential in cellular models using human bone marrow (BM) stromal and mesenchymal stem cells. We hypothesized that the proliferative, migratory, angiogenic and osteoinductive potential of UCBp is derived from cytokines and growth factors associated with the allograft.

- Protein multiplexing based profiling quantified 44 different cytokines in the UCBp conditioned media (CM) which are essential for bone repair and regeneration. These cytokines retained biological activity, as evident from the positive impact of the UCBp-derived CM on cell proliferation and migration of BM-mesenchymal stem cells and BM stromal cells. UCBp-derived CM enhanced cellular VEGF-A secretion, which further induced angiogenesis of primary endothelial cells, suggesting a feedforward mechanism and cross communication among different cell types. Differentiation of BM-MSCs into osteogenic lineage is important for bone formation, and our data demonstrate that administering UCBP-CM significantly shortens the overall time required for such osteogenic differentiation.

- Taken together, the data conclusively establish that UCBp is enriched in cytokines and growth factors that play an important role in bone regeneration and repair. The insight gained from this study elucidates the mechanism of action of UCBp in regulating cellular function and implies a potential clinical application of this allograft in diverse orthopedic disorders. 


\section{Acknowledgments}

OMM received fellowship (Summer 2018) from Idaho INBRE Program which is supported through Institutional Development Awards (IDeA) from the National Institute of General Medical Sciences of the National Institutes of Health under Grant \#P20GM103408. The authors also thank Daniel Thrasher for his excellent editorial assistance in preparation of the manuscript.

\section{Financial \& competing interests disclosure}

MS Sane, N Misra, S Czop, H Tang, CD Jones and S Banerjee Mustafi are employees of Burst Biologics, DBA SMART Surgical Inc, Boise, ID. LT Khoo is a clinical collaborator of Burst Biologics. Burst Biologics has filed a patent pertaining to the processing method of the cellular allograft described in the manuscript. The authors have no other relevant affiliations or financial involvement with any organization or entity with a financial interest in or financial conflict with the subject matter or materials discussed in the manuscript apart from those disclosed.

No writing assistance was utilized in the production of this manuscript.

\section{Ethical conduct of research}

Umbilical cord blood was procured from consenting adults and manufactured in a Food and Drug Administration (FDA) registered facility following all necessary regulatory guidelines as per the FDA's Good Tissue Practice.

\section{Supplementary data}

To view the supplementary data that accompany this paper please visit the journal website at: www.futuremedicine.com/doi/suppl/10.2217/rme-2018-0102

\section{References}

1. Niikura T, Lee SY, Sakai Y, Nishida K, Kuroda R, Kurosaka M. Causative factors of fracture nonunion: the proportions of mechanical, biological, patient-dependent, and patient-independent factors. J. Orthop. Sci. 19(1), 120-124 (2014).

2. Bhandari M, Fong K, Sprague S, Williams D, Petrisor B. Variability in the definition and perceived causes of delayed unions and nonunions: a cross-sectional, multinational survey of orthopaedic surgeons. JBJS 94(15), e109 (2012).

3. Buza Iii JA, Einhorn T. Bone healing in 2016. Clin. Cases Miner. Bone Metab. 13(2), 101 (2016).

4. Lee C, Dorcil J, Radomisli TE. Nonunion of the spine: a review. Clin. Orthop. Relat Res. 419, 71-75 (2004).

5. Dimitriou R, Tsiridis E, Giannoudis PV. Current concepts of molecular aspects of bone healing. Injury 36(12), 1392-1404 (2005).

6. Mckibbin B. The biology of fracture healing in long bones. J. Bone Joint Surg. Br. 60(2), 150-162 (1978).

7. Bruder SP, Fink DJ, Caplan AI. Mesenchymal stem cells in bone development, bone repair, and skeletal regenaration therapy. J. Cell. Biochem. 56(3), 283-294 (1994).

8. Krampera M, Pizzolo G, Aprili G, Franchini M. Mesenchymal stem cells for bone, cartilage, tendon and skeletal muscle repair. Bone 39(4), 678-683 (2006).

9. Einhorn TA. The cell and molecular biology of fracture healing. Clin. Orthop. Relat Res. 355, S7-S21 (1998).

10. Einhorn TA, Gerstenfeld LC. Fracture healing: mechanisms and interventions. Nat. Rev. Rheumatol. 11(1), 45 (2015).

11. Daley GQ. The promise and perils of stem cell therapeutics. Cell Stem Cell 10(6), 740-749 (2012).

12. Kimelman N, Pelled G, Helm GA, Huard J, Schwarz EM, Gazit D. Gene-and stem cell-based therapeutics for bone regeneration and repair. Tissue Eng. 13(6), 1135-1150 (2007).

13. Erices A, Conget P, Minguell JJ. Mesenchymal progenitor cells in human umbilical cord blood. Br. J. Haematol. 109(1), 235-242 (2000).

14. Lee SJ. Classification systems for chronic graft-versus-host disease. Blood 129, 30-37 (2016).

15. Bieback K, Kern S, Klüter H, Eichler H. Critical parameters for the isolation of mesenchymal stem cells from umbilical cord blood. Stem Cells 22(4), 625-634 (2004).

16. Kern S, Eichler H, Stoeve J, Klüter H, Bieback K. Comparative analysis of mesenchymal stem cells from bone marrow, umbilical cord blood, or adipose tissue. Stem Cells 24(5), 1294-1301 (2006).

17. Sibov TT, Severino P, Marti L et al. Mesenchymal stem cells from umbilical cord blood: parameters for isolation, characterization and adipogenic differentiation. Cytotechnology 64(5), 511-521 (2012).

18. Divya MS, Roshin GE, Divya TS et al. Umbilical cord blood-derived mesenchymal stem cells consist of a unique population of progenitors co-expressing mesenchymal stem cell and neuronal markers capable of instantaneous neuronal differentiation. Stem Cell Res. Ther:3(6), 57 (2012).

19. Lee OK, Kuo TK, Chen W-M, Lee K-D, Hsieh S-L, Chen T-H. Isolation of multipotent mesenchymal stem cells from umbilical cord blood. Blood 103(5), 1669-1675 (2004). 
20. Wagner W, Wein F, Seckinger A et al. Comparative characteristics of mesenchymal stem cells from human bone marrow, adipose tissue, and umbilical cord blood. Exp. Hematol. 33(11), 1402-1416 (2005).

21. Newell LF, Flowers ME, Gooley TA et al. Characteristics of chronic GVHD after cord blood transplantation. Bone Marrow Transplant. 48(10), 1285 (2013).

22. Secco M, Zucconi E, Vieira NM et al. Multipotent stem cells from umbilical cord: cord is richer than blood! Stem Cells 26(1), 146-150 (2008).

23. Nagamura-Inoue T, He H. Umbilical cord-derived mesenchymal stem cells: their advantages and potential clinical utility. World J. Stem Cells 6(2), 195 (2014).

24. Garanina EE, Gatina D, Martynova EV, Rizvanov A, Khaiboullina S, Salafutdinov I. Cytokine profiling of human umbilical cord plasma and human umbilical cord blood mononuclear cells. Blood 30(1), 4814 (2017).

25. Takahashi N, Uehara R, Kobayashi M et al. Cytokine profiles of seventeen cytokines, growth factors and chemokines in cord blood and its relation to perinatal clinical findings. Cytokine 49(3), 331-337 (2010).

26. Pereira T, Ivanova G, Caseiro AR et al. MSCs conditioned media and umbilical cord blood plasma metabolomics and composition. PLoS ONE 9(11), e113769 (2014).

27. Bouyou-Akotet MK, Kombila M, Kremsner PG, Mavoungou E. Cytokine profiles in peripheral, placental and cord blood in pregnant women from an area endemic for Plasmodium falciparum. Eur. Cytokine Netw. 15(2), 120-125 (2004).

28. Souayah N, Ende N, Chen H. The cytokine profile of human umbilical cord blood. Neurology 78(1), P02-015 (2012).

29. Edderkaoui B. Potential role of chemokines in fracture repair. doi:10.3389/fendo.2017.00039 (2017) (Epub ahead of print).

30. Son BR, Marquez-Curtis LA, Kucia M et al. Migration of bone marrow and cord blood mesenchymal stem cells in vitro is regulated by stromal-derived factor-1-CXCR4 and hepatocyte growth factor-c-met axes and involves matrix metalloproteinases. Stem Cells 24(5), 1254-1264 (2006).

31. Doi H, Kitajima Y, Luo L et al. Potency of umbilical cord blood-and Wharton's jelly-derived mesenchymal stem cells for scarless wound healing. Sci. Rep. 6, 18844 (2016).

32. Hocking AM. The role of chemokines in mesenchymal stem cell homing to wounds. Adv. Wound Care 4(11), 623-630 (2015).

33. Lin W, Xu L, Zwingenberger S, Gibon E, Goodman SB, Li G. Mesenchymal stem cells homing to improve bone healing. J. Orthop. Transl. 9, 19-27 (2017).

34. Yang C, Yang P. The promotional effect of mesenchymal stem cell homing on bone tissue regeneration. Curr. Stem Cell Res. Ther. 12(5), 365-376 (2017).

35. Yao W, Lay YaE, Kot A et al. Improved mobilization of exogenous mesenchymal stem cells to bone for fracture healing and sex difference. Stem Cells 34(10), 2587-2600 (2016).

36. Wang X, Wang Y, Gou W, Lu Q, Peng J, Lu S. Role of mesenchymal stem cells in bone regeneration and fracture repair: a review. Int. Orthop. 37(12), 2491-2498 (2013).

37. Arican O, Aral M, Sasmaz S, Ciragil P. Serum levels of TNF- $\alpha$, IFN- $\gamma$, IL-6, IL-8, IL-12, IL-17, and IL-18 in patients with active psoriasis and correlation with disease severity. Mediators Inflamm. 2005(5), 273-279 (2005).

38. Chen F-M, Liu X. Advancing biomaterials of human origin for tissue engineering. Prog. Polym. Sci. 53, 86-168 (2016).

39. Solves P, Mirabet V, Planelles D, Carbonell-Uberos F, Roig R. Influence of volume reduction and cryopreservation methodologies on quality of thawed umbilical cord blood units for transplantation. Cryobiology 56(2), 152-158 (2008).

40. Jaime-Pérez JC, Monreal-Robles R, Rodríguez-Romo LN, Mancías-Guerra C, Herrera-Garza JL, Gómez-Almaguer D. Evaluation of volume and total nucleated cell count as cord blood selection parameters: a receiver operating characteristic curve modeling approach. Am. J. Clin. Pathol. 136(5), 721-726 (2011).

41. Naing MW, Gibson DA, Hourd P et al. Improving umbilical cord blood processing to increase total nucleated cell count yield and reduce cord input wastage by managing the consequences of input variation. Cytotherapy 17(1), 58-67 (2015).

42. Nowak-Sliwinska P, Alitalo K, Allen E et al. Consensus guidelines for the use and interpretation of angiogenesis assays. Angiogenesis 1-108 (2018).

43. Marupanthorn K, Tantrawatpan C, Kheolamai P, Tantikanlayaporn D, Manochantr S. Bone morphogenetic protein-2 enhances the osteogenic differentiation capacity of mesenchymal stromal cells derived from human bone marrow and umbilical cord. Int. J. Mol. Med. 39(3), 654-662 (2017).

44. Wagner JE, Barker JN, Defor TE et al. Transplantation of unrelated donor umbilical cord blood in 102 patients with malignant and nonmalignant diseases: influence of CD34 cell dose and HLA disparity on treatment-related mortality and survival. Blood 100(5), 1611-1618 (2002).

45. Dawson G, Sun JM, Davlantis KS et al. Autologous cord blood infusions are safe and feasible in young children with autism spectrum disorder: results of a single-center Phase I open-label trial. Stem Cells Transl. Med. 6(5), 1332-1339 (2017).

46. Harris DT, Rogers I. Umbilical cord blood: a unique source of pluripotent stem cells for regenerative medicine. Curr. Stem Cell Res. Ther. 2(4), 301-309 (2007). 
47. Yagi H, Soto-Gutierrez A, Parekkadan B et al. Mesenchymal stem cells: mechanisms of immunomodulation and homing. Cell Transpl. 19(6-7), 667-679 (2010).

48. Caplan AI. Mesenchymal stem cells: time to change the name! Stem Cells Transl. Med. 6(6), 1445-1451 (2017).

49. Phinney DG, Pittenger MF. Concise review: MSC-derived exosomes for cell-free therapy. Stem Cells 35(4), 851-858 (2017).

50. Stanovici J, Le Nail L-R, Brennan M et al. Bone regeneration strategies with bone marrow stromal cells in orthopaedic surgery. Curr. Res. Transl. Med. 64(2), 83-90 (2016).

51. Grayson WL, Bunnell BA, Martin E, Frazier T, Hung BP, Gimble JM. Stromal cells and stem cells in clinical bone regeneration. Nat. Rev. Endocrinol. 11(3), 140 (2015).

52. Chang F, Steelman L, Lee J et al. Signal transduction mediated by the Ras/Raf/MEK/ERK pathway from cytokine receptors to transcription factors: potential targeting for therapeutic intervention. 17 Leukemia 17, 1263-1293 (2003).

53. Cross MJ, Claesson-Welsh L. FGF and VEGF function in angiogenesis: signalling pathways, biological responses and therapeutic inhibition. Trends Pharmacol. Sci. 22(4), 201-207 (2001).

54. Chang F, Steelman LS, Shelton JG et al. Regulation of cell cycle progression and apoptosis by the Ras/Raf/MEK/ERK pathway. Int. J. Oncol. 22(3), 469-480 (2003).

55. Lin W, Xu L, Zwingenberger S, Gibon E, Goodman SB, Li G. Mesenchymal stem cells homing to improve bone healing. doi:10.1016/j.jot.2017.03.002 (2017) (Epub ahead of print).

56. Ponte AL, Marais E, Gallay N et al. The in vitro migration capacity of human bone marrow mesenchymal stem cells: comparison of chemokine and growth factor chemotactic activities. Stem Cells 25(7), 1737-1745 (2007).

57. Fiedler J, Röderer G, Günther KP, Brenner RE. BMP-2, BMP-4, and PDGF-bb stimulate chemotactic migration of primary human mesenchymal progenitor cells. J. Cell. Biochem. 87(3), 305-312 (2002).

58. Shi M, Li J, Liao L et al. Regulation of CXCR4 expression in human mesenchymal stem cells by cytokine treatment: role in homing efficiency in NOD/SCID mice. Haematologica 92(7), 897-904 (2007).

59. Hu C, Yong X, Li C et al. CXCL12/CXCR4 axis promotes mesenchymal stem cell mobilization to burn wounds and contributes to wound repair. J. Surg. Res. 183(1), 427-434 (2013).

60. Hankenson KD, Dishowitz M, Gray C, Schenker M. Angiogenesis in bone regeneration. Injury 42(6), 556-561 (2011).

61. Carano RA, Filvaroff EH. Angiogenesis and bone repair. Drug Discov. Today 8(21), 980-989 (2003).

62. Kleinheinz J, Stratmann U, Joos U, Wiesmann HP. VEGF-activated angiogenesis during bone regeneration. J. Oral Maxillofac. Surg. doi:10.1016/j.joms.2005.05.303 (2005) (Epub ahead of print).

63. Gerber H-P, Vu TH, Ryan AM, Kowalski J, Werb Z, Ferrara N. VEGF couples hypertrophic cartilage remodeling, ossification and angiogenesis during endochondral bone formation. Nat. Med. 5(6), 623 (1999).

64. Eckardt H, Bundgaard KG, Christensen KS, Lind M, Hansen ES, Hvid I. Effects of locally applied vascular endothelial growth factor (VEGF) and VEGF-inhibitor to the rabbit tibia during distraction osteogenesis. J. Orthop. Res. 21(2), 335-340 (2003).

65. Issbrucker K, Marti HH, Hippenstiel S et al. p38 MAP kinase - a molecular switch between VEGF-induced angiogenesis and vascular hyperpermeability. FASEB J. 17(2), 262-264 (2003).

66. Wang M, Crisostomo PR, Herring C, Meldrum KK, Meldrum DR. Human progenitor cells from bone marrow or adipose tissue produce VEGF, HGF, and IGF-I in response to TNF by a p38 MAPK-dependent mechanism. Am. J. Physiol. Regul. Integr. Comp. Physiol. 291(4), R880-R884 (2006).

67. Boyce BF, Xing L. Functions of RANKL/RANK/OPG in bone modeling and remodeling. Arch. Biochem. Biophys. 473(2), 139-146 (2008).

68. Kohli SS, Kohli VS. Role of RANKL-RANK/osteoprotegerin molecular complex in bone remodeling and its immunopathologic implications. Ind. J. Endocrinol. Metab. 15(3), 175 (2011).

69. Payne K, Erickson C, Shaw N et al. Blockade of osteoclast-mediated bone resorption with a RANKL inhibitor enhances spinal fusion in a rat model. J. Bone Mineral Res. 32, S348-S349 (2017).

70. El Bialy I, Jiskoot W, Nejadnik MR. Formulation, delivery and stability of bone morphogenetic proteins for effective bone regeneration. Pharm. Res. 34(6), 1152-1170 (2017).

71. Rodan GA, Martin TJ. Therapeutic approaches to bone diseases. Science 289(5484), 1508-1514 (2000).

72. Street J, Bao M, Bunting $S$ et al. Vascular endothelial growth factor stimulates bone repair by promoting angiogenesis and bone turnover. Proc. Natl Acad. Sci. 99(15), 9656-9661 (2002).

73. Nakamura T, Hara Y, Tagawa M et al. Recombinant human basic fibroblast growth factor accelerates fracture healing by enhancing callus remodeling in experimental dog tibial fracture. J. Bone Mineral Res. 13(6), $942-949$ (1998).

74. Eggenhofer E, Luk F, Dahlke MH, Hoogduijn MJ. The life and fate of mesenchymal stem cells. Front. Immunol. 5, 148 (2014). 